\title{
Chromium thin-film polarizer for high intensity light
}

\author{
Damian A. Michalik, ${ }^{*}$ Paweł S. Jung, ${ }^{1}$ Bartłomiej W. Klus, ${ }^{1}$ Andrzej Kowalik, ${ }^{2}$ Anna Rojek, ${ }^{2}$ Urszula A. Laudyn ${ }^{1}$ \\ and Mirosław A. Karpierz ${ }^{1}$ \\ ${ }^{1}$ Faculty of Physics, Warsaw University of Technology, Koszykowa 75, 00-662 Warszawa, Poland \\ ${ }^{2}$ Institute of Electronic Materials Technology, Wolczynska 133, 01-919 Warszawa, Poland.
}

Received September 20, 2017; accepted September 27, 2017; published September 30, 2017

\begin{abstract}
In this work, we investigate a thin-film polarizer for a high intensity electromagnetic (EM) beam based on $\mathrm{Cr}$ nano wire arrays. Commonly used thin-film polarizing components are very sensitive for high power of EM waves and can be easily damaged by focused beams. The solution to this problem could be a thin-film polarizer based on metallic subwavelength structures. This type of optical element has huge resistance compared to typical thin-film polarizers. However, designing such an optical element for the proper wavelength of an EM wave and transmission is not an easy task. In this paper we present numerical as well as experimental results for a specially designed chromium thin-film polarizer for a wavelength of $532 \mathrm{~nm}$.
\end{abstract}

Sub-wavelength metallic structures give an opportunity to engineer elements with unusual optical responses that are not available in nature [1-3]. Such metallic structures can be used in thin-film polarizers $[4,5]$. In general, such polarizers consist of a metallic wire grid which could have different shapes to provoke expected interaction between a structure and an incident light wave. The patent concerning a wire grid polarizer was reported in the United States in 2000 [6]. It was a simple idea with an important impact worldwide on metallic structures such as polarizers. This work changed the point of view on small-scale metal wire grid structures with respect to plasmonic effects.

The main purpose of this work was to use the current state of knowledge to design and test a new simple thinfilm polarizer based on $\mathrm{Cr}$ nano wire arrays for high intensity light beams of a $532 \mathrm{~nm}$ wavelength. Moreover, in this work we introduce the physical background needed to design such a polarizer.

The scheme of our polarizer is presented in Fig. 1. The main element is a planar array of metallic wires with a rectangular shape, where the main parameters of our structure is a single wire with width $x$, thickness $y$ and array period $\Lambda$. An incident electromagnetic wave on the polarizer is partially reflected, absorbed and transmitted. The proportions depend on EM wave polarization as well as on structure parameters and are correlated with interface conditions of Maxwell's equations:

$$
\boldsymbol{n}_{12} \times\left(\boldsymbol{E}_{1}-\boldsymbol{E}_{2}\right)=0
$$

*E-mail: michalik.damiann@gmail.com

$$
\begin{gathered}
\boldsymbol{n}_{12} \cdot\left(D_{1}-D_{2}\right)=\rho_{s} \\
\boldsymbol{n}_{12} \times\left(H_{1}-H_{2}\right)=0 \\
\boldsymbol{n}_{12} \cdot\left(\boldsymbol{B}_{1}-\boldsymbol{B}_{2}\right)=j_{s}
\end{gathered}
$$

where $\boldsymbol{n}_{\mathbf{1 2}}$ is the normal vector from medium 1 to medium $2, \rho_{s}$ is the surface charge between the media, $j_{S}$ is the surface current density between the two media.

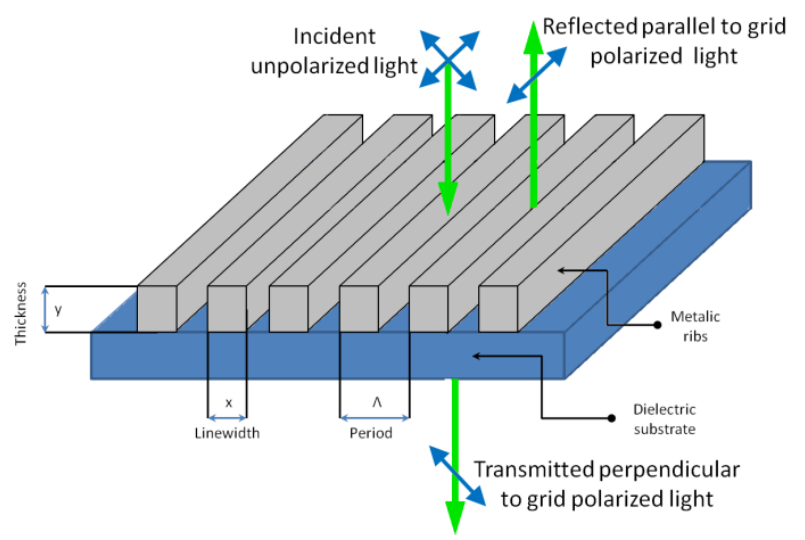

Fig. 1. The schematic diagram of a thin-film polarizer.

It is possible to linearly polarize an electric field perpendicular to the wires using this type of structures. That is the idea of any polarizer, however metallic ribs transfer heat rather than typical polarizers so it is a more appropriate solution for high intensity beams. By choosing materials and proper structure parameters of line width $x$, thickness $y$ and period $\Lambda$, it is possible to make efficient polarizers for a given wavelength of light.

To understand better and get deeper into the physics of our polarizers we used the Finite Difference Time Domain method (FDTD) [7]. To accelerate numerical simulations we simplified our analyzed structure to $2 \mathrm{D}$ by neglecting the dimension associated with the length of polarizer ribs. It could be done because our polarizer had a planar configuration and the length of polarizer ribs (about $5 \mathrm{~mm}$ ) was much bigger compared to launched beam waist $(10 \mu \mathrm{m})$. In FDTD simulations we used 
Perfectly Matched Layers (PML) [8] to minimize reflections from the computational window and the $\mathrm{Yu}-$ Mittra method [9] to minimize the error associated with approximation of a used finite rectangular grid. In numerical simulations we used an EM wave of $532 \mathrm{~nm}$

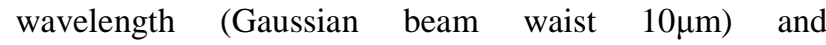
corresponding chromium electric permittivity: $\varepsilon=$ $-11.31+25.72 i$ [9]. In further analyses we will use the following signs: $90^{\circ}$ for $\mathrm{EM}$ waves with polarization perpendicular to metallic polarizer ribs and $0^{\circ}$ for parallel. An example of interaction between an incident light wave and our structure for both polarizations is presented in Fig. 2. As we can see for polarization $0^{\circ}$ of the EM wave, most of it is absorbed and reflected by the metallic ribs (Fig. 2a), while for polarization $90^{\circ}$ the EM wave is guided between the ribs. On the other hand, for polarization $90^{\circ}$ out of plasmonic resonance (Fig. 2c) we can observe both mentioned phenomena.
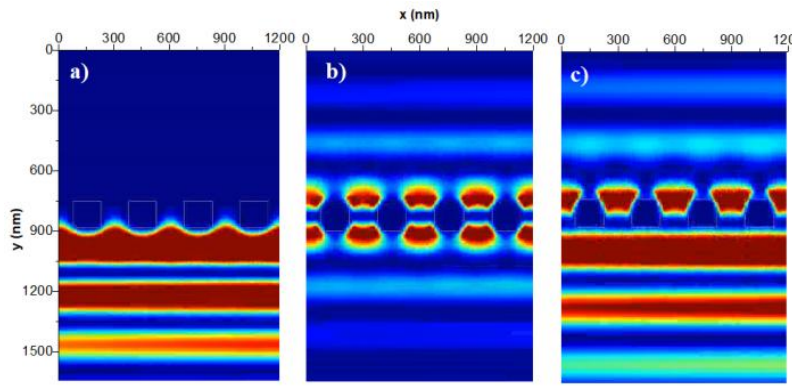

Fig. 2. An example of EM field propagation (Pointing vector) for input Gaussian beam $w_{o}=10 \mu \mathrm{m}$ and polarization a) $0^{\circ}$, b) $90^{\circ}$ through $\mathrm{Cr}$ thin-film polarizer with parameters $x=150 \mathrm{~nm}, y=150 \mathrm{~nm}$ and $\Lambda=300 \mathrm{~nm}$ and c) $90^{\circ}$ out of plasmonic resonance $(y=130 \mathrm{~nm})$.

In detail we checked numerically the influence of characteristic parameters of our polarizer on a transmission incident EM wave. The first numerical results of wave transmittance for various thickness $y$ and fixed width $x=200 \mathrm{~nm}$ and period $\Lambda=300 \mathrm{~nm}$ are presented in Fig. 3. In the figure, the black curve represents an EM wave perpendicular to the ribs and the red one - parallel (the same for other graphs). As we could expect, the EM wave polarized parallel to the ribs (red curve) was blocked and the perpendicular polarized wave was partially transmitted. The value of transmittance depends highly on thickness. Moreover, in the graph for one polarization (Fig. 4 black line) we see characteristic plasmonic resonance peaks. We investigated the impact of the period $\Lambda$ for the fixed width $x=200 \mathrm{~nm}$ and the thickness $y=100 \mathrm{~nm}$. These results are presented in Fig. 4 . As it can be expected, for the periods below the EM wavelength $(\Lambda<\lambda)$ our polarizer starts to work as a subwavelength metallic thin-film polarizer. While for periods greater than the wavelength, the polarizer just works as a diffraction grating. The last thing we numerically investigated was the influence of various widths $x$ with the constant period $\Lambda=300 \mathrm{~nm}$ and the thickness $y=100 \mathrm{~nm}$ on transmittance. Our collective results are presented in Fig. 5. Form this analysis it is seen that by choosing the proper line width we can easily play with transmittance for both polarizations.

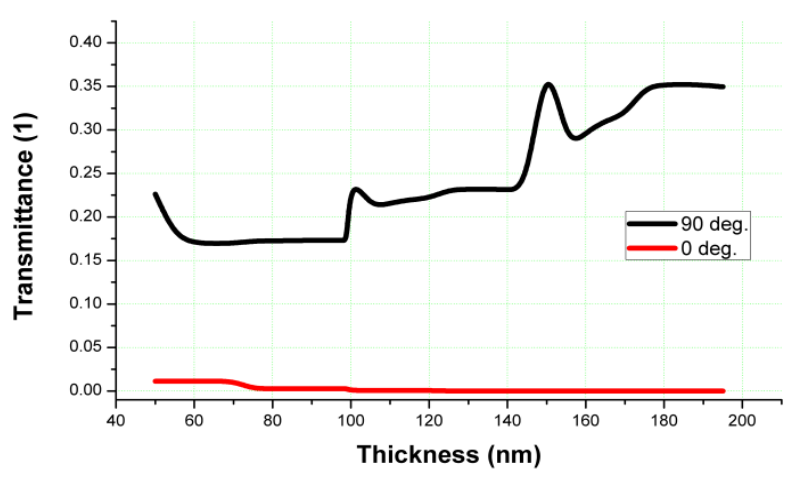

Fig. 3. Chromium thin-film polarizer transmittance chart dependent on thickness while $x=200 \mathrm{~nm}$ and $\Lambda=300 \mathrm{~nm}$.

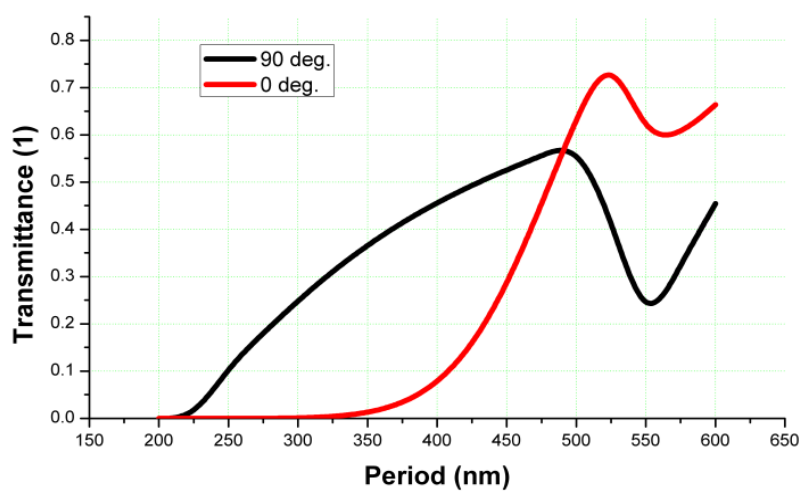

Fig. 4. Chromium thin-film polarizer transmittance chart dependent on period while $x=200 \mathrm{~nm}$ and $y=100 \mathrm{~nm}$.

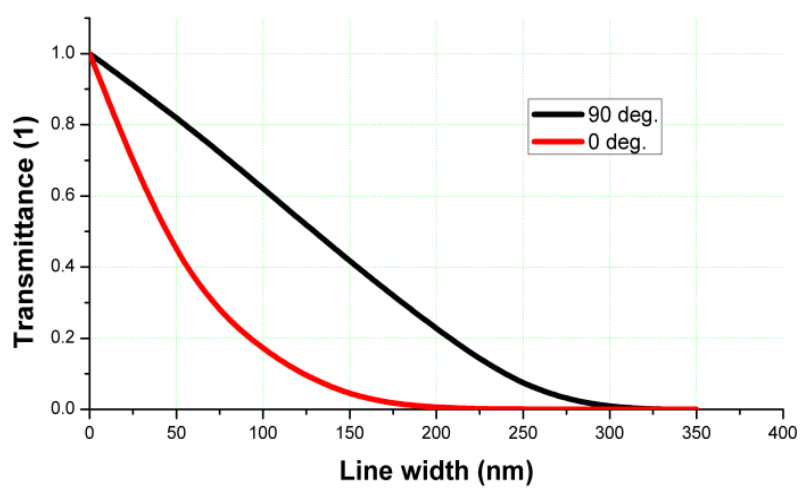

Fig. 5. Chromium thin-film polarizer transmittance chart dependent on line width while $y=100 \mathrm{~nm}$ and $\Lambda=300 \mathrm{~nm}$.

Finally, based on our numerical simulation and technical possibilities we made 3 prototypes of our 
chromium thin-film polarizers with the following parameters:

- TFP100: $x=100 \mathrm{~nm}, y=100 \mathrm{~nm}, \Lambda=300 \mathrm{~nm}$,

- TFP150: $x=150 \mathrm{~nm}, y=100 \mathrm{~nm}, \Lambda=300 \mathrm{~nm}$,

- TFP200: $x=200 \mathrm{~nm}, y=100 \mathrm{~nm}, \Lambda=300 \mathrm{~nm}$.

Those polarizers were fabricated using electron-beam lithography. Unfortunately, our experimental structures are not perfect and the above parameters are only indicative. Therefore we can expect some discrepancies with numerical results. The transmittance capacity of an EM wave for our polarizers was experimentally examined in the setup presented in Fig. 6. In our experiment we used, among others, a light source for wavelength $532 \mathrm{~nm}$ from Spectra Physics Millennia as well as a GlanThomson polarizer, Thorlabs half-wave Plate and an Ophir Nova II Laser Power Meter.

Our experimental results are presented in Fig. 7, which shows the dependence of transmittance versus the angle of polarization of an input Gaussian beam with a power of $500 \mathrm{~mW}$ for three polarizers.

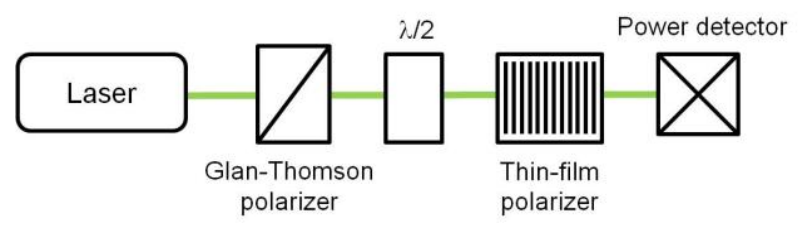

Fig. 6. The experimental setup.

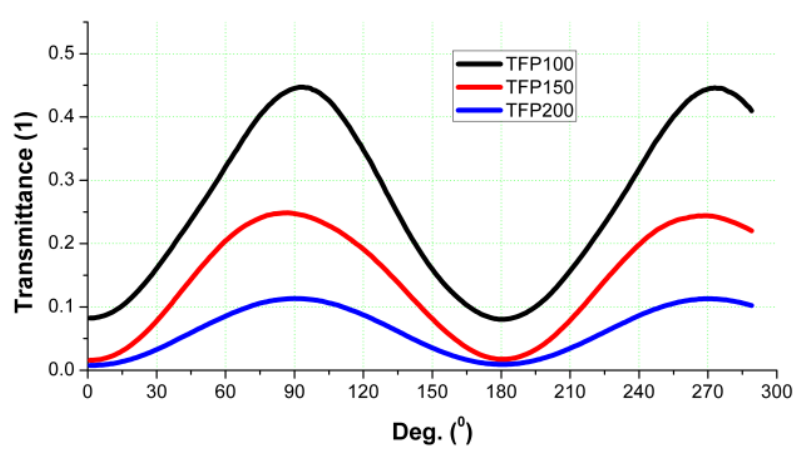

Fig. 7. The experimental results for three thin-film polarizers TF100, TFP150 and TFP200.

Tab. 1. The comparative results from numerical simulations and experiment.

\begin{tabular}{|c|c|c|c|c|}
\hline \multirow{2}{*}{ Polarizer } & \multicolumn{3}{|c|}{ Transmittance values } \\
\cline { 2 - 5 } & \multicolumn{2}{|c|}{ Theoretical } & \multicolumn{2}{c|}{ Experimental } \\
\cline { 2 - 5 } & $0^{\circ}$ & $90^{\circ}$ & $0^{\circ}$ & $90^{\circ}$ \\
\hline TFP100 & 0.1549 & 0.6217 & 0.0824 & 0.4721 \\
\hline TFP150 & 0.0291 & 0.4143 & 0.0159 & 0.2475 \\
\hline TFP200 & 0.0022 & 0.2244 & 0.0075 & 0.1182 \\
\hline
\end{tabular}

The experimental results of transmittance in the graph behave like a cosine function, which corresponds to Malus's law. It has to be noticed that TF100 has the widest transmission gap and, unfortunately, higher than the zero blocking property. Other results of TFP150 and TFP200 offer strong possibilities of blocking a parallel polarized wave but fairly small passing transmission values. The best transmission results has TFP100 because it has the widest period gap value but its blocking possibility is faint due to too a small line width. This kind of behavior is confirmed in theoretical analysis. In Tab.1 the comparative results for numerical simulations and the experiment are presented. As it can be noticed, the theoretical transmission values from numerical simulations are fairly higher. This is the result of at least two factors. The first is correlated with fabrication process accuracy which results in our polarizer parameters not being the same in the whole volume. And the second one is correlated with theoretical calculations where a dielectric substrate was neglected.

Summarizing, we investigated a specially designed (by us) thin-film chromium polarizer for a of wavelength $532 \mathrm{~nm}$, resistant to a high intensity EM wave. Our experimental results are in fairly good agreement with numerical simulations. The transmittance efficiency of our polarizer could be optimized by using numerical results. One of the possibilities could be increasing the thickness of polarizers ribs. Unfortunately, this possibility depends also on the fabrication process, which will be investigated in future.

This work was supported by National Center for Research and Development by the grant agreement DOB$1-6 / 1 / P S / 2014$.

\section{References}

[1] W. Zhou, K. Li, C. Song, P. Hao, M. Chi, M. Yu, Y. Wu, Opt. Expr. 23, 11 (2015).

[2] W. L. Barnes, A . Dereux, T.W. Ebbesen, Nature 424, 824 ( 2003).

[3] C. Lee, E. Sim, D. Kim, Opt. Expr. 25, 7 (2017).

[4] A. Lehmuskero, Metallic thin film structures and polarization shaping gratings (University of Eastern Finland 2010).

[5] Y. Leroux, J.C. Lacroix, C. Fave, V. Stockhausen, N. Felidj, J. Grandm, A. Hohenau, J.R. Krenn, Nano Lett. 5, 9 (2009).

[6] R.T. Perkins, D.P. Hansen, E.W. Gardner, J.M. Thorne, A.A. Robbins, Broadband wire grid polarizer for the visible spectrum, US 6122103 (2000).

[7] D.M. Sullivan, Electromagnetic simulation using the FDTD method (New York: IEEE Press Series 2000).

[8] J.P. Berenger, Perfectly Matched Layer (PML) for Computational Electromagnetics (Morgan and Claypool Publishers 2007).

[9] W. Yu, R. Mittra, IEEE Antennas Propagat. Magaz. 42(5), 28 (2000).

[10] L.W. Bos, D.W. Lynch, Phys. Rev. Sect. B, 2, 4267 (1970). 